\title{
Cost-Efficient RAA Technology for Development of the High-Gain Steerable Antennas for mmWave Communications
}

\author{
Olesya BOLKHOVSKAYA ${ }^{\mathrm{a}, 1}$ Alexander MALTSEV ${ }^{\mathrm{a}, \mathrm{b}}$, Valentin SELEZNEV ${ }^{\mathrm{a}}$ \\ and Ilya BOLOTIN ${ }^{\mathrm{c}}$

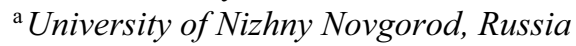 \\ ${ }^{\mathrm{b}}$ LG Corp, Russia. \\ 'Intel Corp, Russia
}

\begin{abstract}
For the future millimeter wave wireless systems the high gain steerable antennas are the key technology to overcome large path loss and support users' mobility and reconfigurable backhauling. This paper introduces a specially designed reflect-array antenna (RAA), integrating single phased antenna array (PAA) module (as a feed source) and flat reflecting surface. The RAA provides high antenna gain by focusing the beam in vertical plane, but, at the same time, supporting beamsteering capability in the horizontal plane. The RAA technology allows creation of the very larger aperture antennas with simplified light-weight design. Two RAA prototypes with different feed source mounting demonstrated reliable multi-gigabit IEEE 802.11 ad link 2.3-4.62 Gbps at distances $100-150 \mathrm{~m}$. These properties of the RAAs make them suitable for both millimeter-wave mobile access and reconfigurable backhauling applications.
\end{abstract}

Keywords. IEEE 802.11ay, mmWave, reflect-array antenna, RAA, 60GHz, beamsteering, $5 \mathrm{G}$

\section{Introduction}

For satisfying the throughput requirements of the $5 \mathrm{G}$ system the future communication systems should exploit all possibilities, including the densification of base stations with usage of heterogeneous networks (HetNets) architecture, massive MIMO, MU-MIMO and CoMP (Coordinated Multi-Point) techniques [1]-[3] and, finally, the bandwidth increase. Millimeter wave communications allow realizing all these possibilities exploiting signals with very large bandwidth and highly directional antennas with narrow beams [3]-[5].

However, for mobile millimeter wave access and reconfigurable backhauling the narrow beam high-gain antennas must be also steerable. During the joint EU-Japan FP7 project MIWEBA [6]-[9], new antenna technologies, the modular antenna array (MAA) and the lens-array antenna (LAA), were proposed to meet these requirements. The MAA consists of multiple independent phased antenna array (PAA) modules (or subarrays), each with its own phase-shifting circuitry and RF part. The modules are

${ }^{1}$ Corresponding Author: Olesya Bolkhovskaya, University of Nizhny Novgorod, Gagarina ave,23, 603109 Nizhny Novgorod, Russia; E-mail:obol@rf.unn.ru 
connected to a common baseband, and each module can act as a single independent phased array. This design has only light limitations on the array adaptability, and degradation in comparison with ideal fully adaptive array (FAA) is not significant [10].

The different path to develop high gain steerable antennas is to combine a single, relatively small, phased antenna array (PAA) with a passive beam focusing system. The LAA is an example of such system, wherein the special dielectric lenses [11]-[12] integrated with a small PAA are used by focusing beam in vertical plane and support the full steering possibility in horizontal plane. Corresponding combined antenna system will not be as flexible and versatile as FAA or MAA, but it can provide a costefficient technique to design high-gain antennas with limited possibilities for beamsteering.

In this work we consider one more approach for developing the high-gain steerable antennas for mmWave communications. This is the reflect-array antenna (RAA) technology [13]-[16] which is using the reflecting surface with special properties for the reflected beam focusing in one plane (in elevation) and allows beam sweeping in another one (in azimuth).

The RAA and LAA have high gain and simultaneously provide beamsteering in a large azimuth angular sector. Of course, both these techniques may be recommended for usage in millimeter wave $5 \mathrm{G}$ communication systems, but the RAA technology has an advantage over the LAA for large aperture antennas, due to very low cost and light weight of the flat passive beam focusing system which may be mounted on any wall in outdoor and indoor environments.

\section{RAA Prototype}

This section describes a design of the reflect-array antenna (RAA) prototype integrating single PAA module and the reflecting flat surface with special properties.

\subsection{Phased Antenna Array Module}

PAA uses a 16-element mmWave rectangular slit loop antenna array in the $60 \mathrm{GHz}$ band, developed by Intel and outlined in [17]. The considered construction geometry and its system of axes are shown in figure 1. The PAA has $2 \times 8$ elements spaced by 0.5 wavelength $(2.5 \mathrm{~mm})$ distance in vertical and horizontal dimensions. Each element has a polarization with an E-field vector parallel to the short side of the array and a radiation pattern close to omni. Full size is $\mathrm{W}=2 \mathrm{~mm}$ width, $\mathrm{H}=9 \mathrm{~mm}$ height and $\mathrm{L}=25 \mathrm{~mm}$ length.

In the developed RAA prototypes the small PAA with 16 antenna elements was used as a primary source. This rectangular (2x8 geometry) PAA was designed by Intel for exploiting in Wi-Fi (IEEE802.11ad) systems in $60 \mathrm{GHz}$ band [17]. The PAA geometry and related system of coordinates are shown in figure 1 . The distance between antenna elements was $0.5 \lambda(2.5 \mathrm{~mm})$. Each element had a vertical polarization and almost omni radiation pattern. 


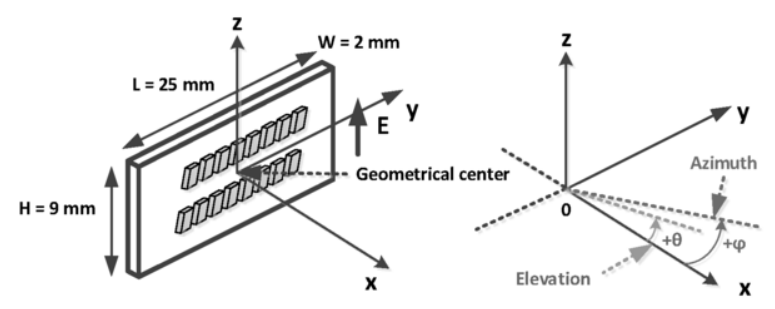

Figure 1. Phased antenna array geometry and related system of coordinates.

The main lobe of the PAA had $14.0^{\circ}$ Half Power Beamwidth (HPBW) in azimuth, and $41.0^{\circ} \mathrm{HPBW}$ in elevation. The total transmit power of the PAA was $\mathrm{P}_{\mathrm{PAA}}=10 \mathrm{dBm}$ and the antenna gain was near to $\mathrm{G}_{\mathrm{PAA}}=15.0 \mathrm{dBi}$.

\subsection{Reflect Array Design}

The RAA uses the similar principle of work as the LAA - focusing the small PAA module beam in the elevation plane, while keeping it almost unchanged (and thus, providing beamsteering capabilities) in the azimuthal plane (see Figure 2). Reflector array of the RAA consists of a number of small phase-shifting reflectors that rotate the phases of the incident wavefront. Usually elementary reflector consists of micro strip patch located at small distances from the metal ground plane (see Figure 3).
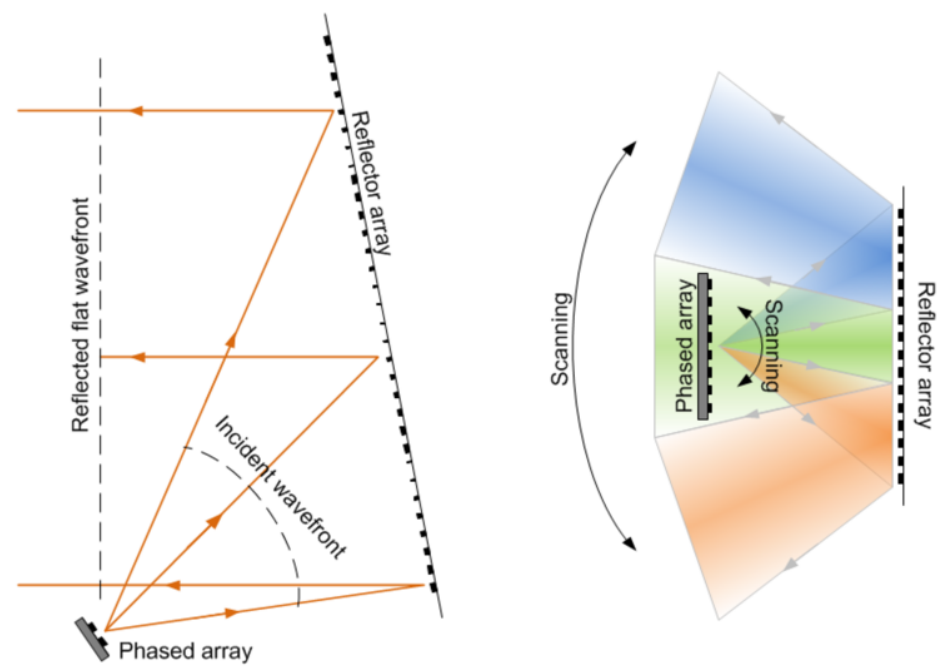

Figure 2. RAA principle illustration. 


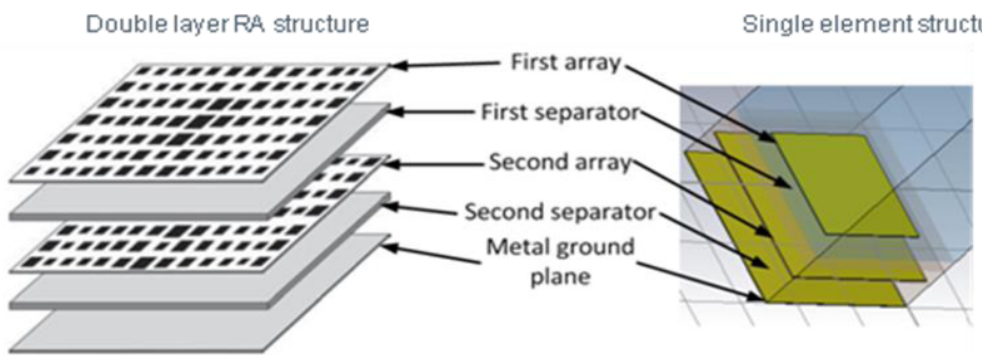

Figure 3. Reflector array structure.

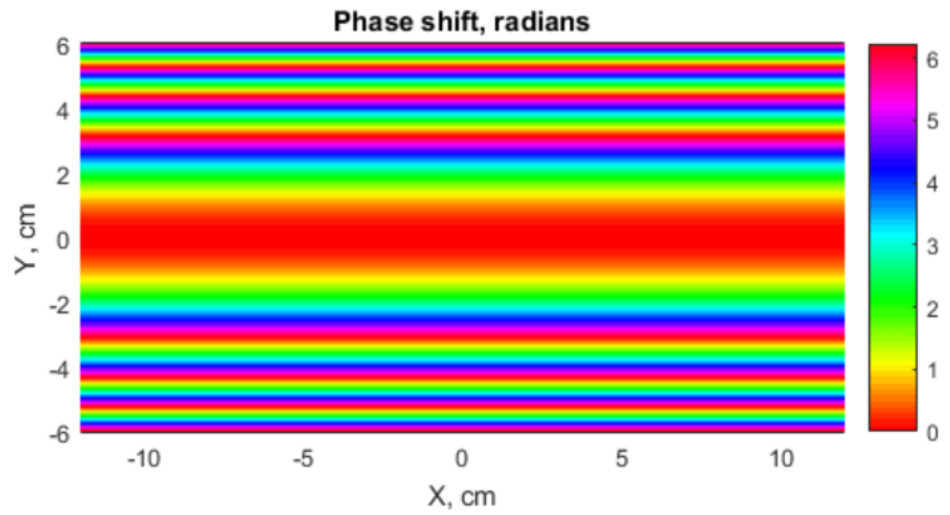

Figure 4. Phase-rotation values distribution for the developed reflector array.

The wave front phase rotation at the given value is setting by patches size and multi-layer structure parameters of the reflector. In the developed reflector array the double layer square patches were used as elementary reflectors (see Figure 3). Usage of the two-layer patch structure allows to obtain the smoother "phase-patch size" dependence, expand the range of possible phase rotations to values greater than $360^{\circ}$ and increases the RAA frequency range up to $10 \%$ of the carrier frequency. By simple adjusting the patches sizes the reflector array can be configured to behave like any type of "curve mirror". For the steerable in azimuth plane RAA the cylindrical-parabolic mirror representation was realized to simplify design of the reflecting surface (see Figure 4).

\subsection{Reflect Array Antenna integration}

Like conventional reflector antennas, axial and offset configurations are available for placement of the feeding PAA module in front of reflector array. Correspondingly, two RAA prototypes were implemented and experimentally investigated - RAA 1 with axial mount and RAA 2 with offset mount of the PAA module, see Figure 5. 



Figure 5. Two RAA prototypes external view

The main characteristics of these prototypes are provided in Table 1.

Table 1. Two RAA prototypes parameters and characteristics.

\begin{tabular}{ccc}
\hline Parameter & Axial feed position & Offset feed position \\
\hline Size & $187 \times 237 \times 1 \mathrm{~mm}$ & $84 \times 154 \times 1 \mathrm{~mm}$ \\
Focus & $200 \mathrm{~mm}$ & $200 \mathrm{~mm}$ \\
Antenna gain & $15+9.5=24.5 \mathrm{dBi}$ & $15+11=26 \mathrm{dBi}$ \\
Azimuth HPBW & $18^{\circ}$ & $14^{\circ}$ \\
Elevation HPBW & $2^{\circ}$ & $2^{\circ}$ \\
TX power & $10 \mathrm{dBm}$ & $10 \mathrm{dBm}$ \\
Operational range & $100-150 \mathrm{~m}$ & $100-150 \mathrm{~m}$ \\
Peak data rate & $4.62-2.3 \mathrm{Gbps}$ & $4.62-2.3 \mathrm{Gbps}$ \\
\hline
\end{tabular}

\section{RAA Field Tests}

\subsection{Experimental Measurement Setup}

Experimental measurements of the developed RAA prototypes antenna patterns and gains were carried out by using a special experimental setup mounted at the rooftops of two neighboring buildings at the University of Nizhny Novgorod campus at $30 \mathrm{~m}$ distance between TX and RX sides (see Figure 6). 


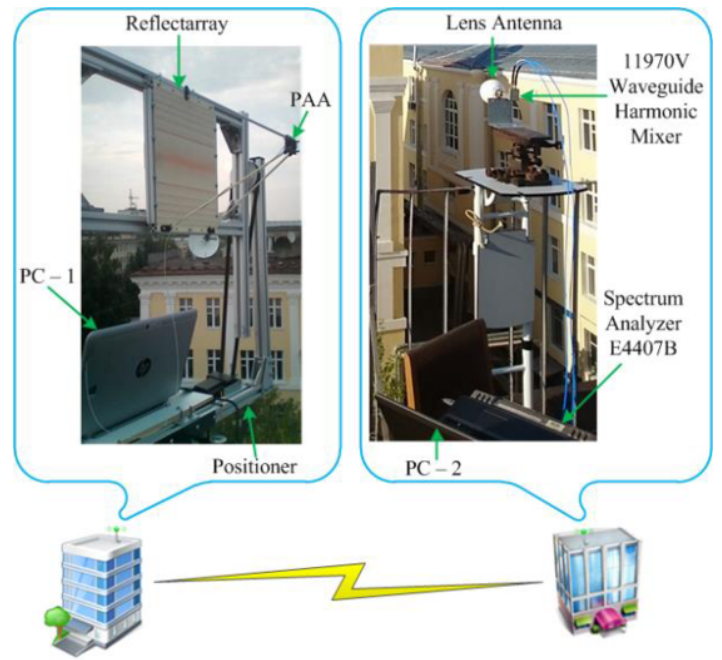

Figure 6. Experimental setup for RAA prototypes characteristics measurement.

At the transmitter side the RAA was mounted at the positioner that performed antenna rotation with angular resolution $\sim 0.4^{\circ}$ in horizontal plane and $0.1^{\circ}$ in vertical plane. The RAA transmitted IEEE 802.11ad waveform signal with central frequency 60 $\mathrm{GHz}$ and bandwidth about $2 \mathrm{GHz}$.

At RX side the special high directional laboratory lens antenna with axial symmetry and aperture size (diameter) equal to $100 \mathrm{~mm}$, HPBW equal to $3.0^{\circ}$, and antenna gain $34.5 \mathrm{dBi}$ was used, to increase SNR and avoid spurious reflections. The RX lens antenna had static position and was perfectly aligned with the TX RAA.

The signal at the RX antenna output was fed to the frequency down converter $11970 \mathrm{~V}$ of Agilent Technologies input and then its power and spectrum were measured by universal spectrum analyzer E4407B of Agilent Technologies. The RX setup performed signal power measurements with start and stop frequencies 59.4 and 61.56 $\mathrm{GHz}$ accordingly with the signal bandwidth $2.16 \mathrm{GHz}$. The sweep time was set equal to $26.0 \mathrm{~ms}$, both resolution and video bandwidths were set equal to $3.0 \mathrm{MHz}$. The positioner and all measurement equipment were controlled remotely via a Bluetooth wireless channel by the software program on the PC.

\subsection{Experimental Results}

The RAA 1 and RAA 2 antenna patterns measurement results in elevation and in azimuth planes are shown in Figure 7. It can be seen that both RAA prototypes are well focusing the power, radiated by the PAA, in the elevation plane, forming the main lobe with HPBW about of 2 degrees. The antenna gain of RAA 1 was about 1.5-2.0 dB less than for RAA 2 that may be obviously explained by the shadowing effect due to the axial position of the PAA. The main lobe beam widths in the azimuth plane a little different for RAA 1 and RAA 2. For RAA 1 with axial feed, it is about $14^{\circ}$, and for RAA 2 with offset feed, it is about $16^{\circ}$. It can be seen that in azimuth plane the radiation pattern have almost the same HPBW as the single PAA, at the same time, in 
the vertical plane the beam is focused, producing large antenna gain and narrow main lobe of the antenna pattern.


Figure 7. Antenna patterns measurement results for RAA 1 with axial feed and RAA 2 with offset feed: a -in elevation plane for azimuth sector $0 ; \mathrm{b}$ - in azimuth plane for different sectors.

To evaluate the RAA prototypes beamsteering capabilities, the beam of the PAA feeding module was directed to three azimuthal sectors at $0^{\circ},+10^{\circ},-10^{\circ}$ (see Figure 7). It can be seen, that for these sectors the antenna gains are practically constant, about $24.5 \mathrm{~dB}$ for the RAA 1 and $26 \mathrm{~dB}$ for the RAA 2. It produces the effective azimuth half power steering range $\left[-15^{\circ}: 15^{\circ}\right]$. But for larger beam deflections the RAAs antenna gains quickly degrades. It is clear, that the cause of this effect is not full power reflection of the steered PAA's beam, due to the limited width of the reflecting surface in horizontal plane. In next generation of the RAA prototypes, this drawback will be fixed. However, even in this first setup, the RAAs allow achieving total 25-26 dBi gain with a limited beamsteering sector, but which is enough, for example, for electronic beam alignment of the backhaul links. If used with 802.11ad standard devices, such TX-RX antenna systems allow transmission at gigabit data rates $4.62-2.3$ Gbps up to 150-200m distance.

\section{Conclusion}

In this paper we have considered a new approach to the design of the high-gain beam steerable antennas for millimeter wave communication systems. The proposed reflectarray antenna (RAA) technology is using the reflecting surface with special properties for the beam focusing in one plane and allow beam sweeping in another one. Theoretical investigations and direct prototyping have proven the efficiency of the proposed RAA technology. Two RAA prototypes with different PAA module mounting were developed and demonstrated the characteristics which provide reliable multi-gigabit link at distances $100-150 \mathrm{~m}$. The RAA technology with flat and lightweight reflecting surfaces allows in cost-efficient way to create mmWave antennas with larger aperture to further increase the antenna gain, beam steering capabilities and achievable transmission range. 


\section{References}

[1] F. Boccardi, R.W. Heath, A. Lozano, T.L. Marzetta and P. Popovski, Five disruptive technology directions for 5G, IEEE Communications Magazine, 5 (2014), 74-80.

[2] A. Davydov, G. Morozov, I. Bolotin and A. Papathanassiou, Evaluation of Joint Transmission CoMP in C-RAN based LTE-A HetNets with large coordination areas, 2013 IEEE Globecom Workshops (GC Wkshps), (2013), 801-806.

[3] R. Heath. Jr, Role of MIMO Beyond LTE: Massive? Coordinated? mmWave?, Workshop on Beyond 3GPP LTE-A ICC, (2013).

[4] E. Torkildson, U. Madhow and M. Rodwell, Indoor Millimeter Wave MIMO: Feasibility and Performance, IEEE Transactions on Wireless Communications, 10 (2011), 4150-4160.

[5] T.S. Rappaport et al., Millimeter Wave Mobile Communications for 5G Cellular: It Will Work!, IEEE Access, 1 (2013), 335-349.

[6] R.J. Weiler et al., Enabling 5G backhaul and access with millimeter-waves, 2014 European Conference on Networks and Communications (EuCNC), 2014, 1-5.

[7] MiWEBA Project homepage http://www.miweba.eu,(FP7-ICT-2013-EU-Japan, project number: 608637, 2013.

[8] ICT FP7 MiWEBA project \#608637, Deliverable D5.3 - Highly-directional steerable mm-wave antennas prototype, http://miweba.eu.

[9] A. Maltsev, A. Sadri, A. Pudeyev and I. Bolotin, Highly Directional Steerable Antennas: High-Gain Antennas Supporting User Mobility or Beam Switching for Reconfigurable Backhauling, IEEE Vehicular Technology Magazine, 11 (2016), 32-39.

[10] A. Maltsev et al., Partially adaptive arrays application for MU-MIMO mode in a MmWave small cells, 2015 IEEE 26th Annual International Symposium on Personal, Indoor, and Mobile Radio Communications (PIMRC), 2015, 315-319.

[11] A. Maltsev, A. Lomayev, A. Pudeyev, I. Bolotin, O. Bolkhovskaya and V. Seleznev, Millimeter-wave Toroidal Lens-Array Antennas Experimental Measurements, 2018 IEEE International Symposium on Antennas and Propagation \& USNC/URSI National Radio Science Meeting, (2018), 607-608.

[12] O. Bolkhovskaya, V. Seleznev, A. Rulkov and A. Maltsev, Steerable Bifocal Lens-Array Antenna at 57-64 GHz, 2019 Conference on Microwave Techniques (COMITE), (2019), 1-6.

[13] J.A. Encinar, Design of two-layer printed reflectarrays using patches of variable size, IEEE Transactions on Antennas and Propagation, 49 (2001).

[14] T. Visentin, W. Keusgen, R. Weiler, Dual-polarized square-shaped offset-fed reflectarray antenna with high gain and high bandwidth in the $60 \mathrm{GHz}$ domain, Proceeding of the 2015 9th European Conference on Antennas and Propagation (EuCAP) (2015).

[15] Wenting Li ; Steven Gao ; Long Zhang ; Qi Luo ; Yuanming Cai An Ultra-Wide-Band Tightly Coupled Dipole Reflectarray Antenna, IEEE Transactions on Antennas and Propagation, 66 (2018), 533-540.

[16] Fan Yang, Ruyuan Deng, Shenheng Xu, Maokun Li Design and Experiment of a Near-Zero-Thickness High-Gain Transmit-Reflect-Array Antenna Using Anisotropic Metasurface, IEEE Transactions on Antennas and Propagation, 66 (2018).

[17] H. K. Pan, B. D. Horine, M. Ruberto, S. Ravid, Mm-Wave Phased Array Antenna and System Integration on Semi-Flex Packaging, IEEE AP-S Conference on Antenna and Propagation for Wireless Communications, (2011). 